\title{
Thermal Treatment Options for Benign Thyroid Nodules-The Role of Radio-Frequency Ablation and Laser Therapy
}

\section{Erivelto Volpi}

\author{
Head and Neck Surgery, Oncology Center, Oswaldo Cruz German Hospital, CETRUS Medical School, São \\ Paulo, Brazil
}

Review of: Bernardi S, Giudici F, Cesareo R, Antonelli G, Cavallaro M, Deandrea M, Giusti M, Mormile A, Negro R, Palermo A, Papini E, Pasqualini V, Raggiunti B, Rossi D, Sconfienza LM, Solbiati L, Spiezia S, Tina D, Vera L, Stacul F, Mauri G 2020 Five-year results of radiofrequency and laser ablation of benign thyroid nodules: A multicenter study from the Italian Minimally Invasive Treatments of the Thyroid Group. Thyroid 30:1759-1770. PMID: 32578498.

\section{SUMMARY}

\section{Background}

Thyroid nodules are prevalent and can be found by palpation in up to $8 \%$ of the population and by ultrasonography in at least 10 to $41 \%$, with this latter rate even higher among elderly patients. Even though the vast majority of these nodules are benign, some may require treatment because of cosmetic reasons or for discomfort or other subjective symptoms of compression to the anterior neck (1).

Radiofrequency ablation (RFA) and laser ablation (LA) are-among other options, such as microwave ablation or high-intensity focused ultrasound-the most common percutaneous minimally invasive techniques using ultrasound guidance to treat benign thyroid nodules (2). However, one of the most undesirable events is the thyroid nodule's regrowth after such treatments (3). A recent multicenter study highlights the experience of the Italian Minimally Invasive Treatments of the Thyroid Group summarizing the use of RFA and LA for the treatment of benign thyroid nodules (4).

\section{Methods}

This is an elegant multicenter retrospective study of eight centers in Italy that specialize in the treatment of thyroid diseases. The primary outcome was to describe the efficacy, regrowth, and retreatment rates of benign thyroid nodules followed for 5 years after thermal ablation therapy (RFA and LA). The secondary outcome was to ascertain the predictive factors for the therapies' effectiveness, nodule regrowth, and need for retreatment. Inclusion criteria were patients with benign diagnostic thyroid nodule cytology, no history of prior thermal ablative treatments to the thyroid nodule, and availability of at least 5 years of follow-up after the thermal ablation.

Authors collected data regarding patients' age, sex, year of treatment, type of procedure, energy delivered (in Joules), baseline nodule volume (in milliliters), nodule structure, nodule function status, nodule volume (in milliliters) 1, 2, 3, 4, and 5 years after the procedure, symptom relapse, type of retreatment, final pathology (in case of surgery), and 

Nodules-The Role of Radio-Frequency Ablation and Laser Therapy

nodule volume (in milliliters) after a second thermal ablation. For patients who received LA of their thyroid nodules, the number of fibers was recorded and the type of RFA electrode was recorded. Nodule volume was measured by ultrasound examination. Thyroid function was assessed with serum thyrotropin (TSH), free triiodothyronine, and free thyroxine concentrations; for those with TSH levels $<0.4 \mu \mathrm{U} /$ $\mathrm{ml}$, thyroid scintingraphy was also performed.

A meticulous statistical analysis was done to compare the results of patients treated with RFA and LA. A sensitivity analysis using propensity-score matching with the $\mathrm{R}$ package Matchlt was performed to control potential confounders and selection bias to compare the two methods of treatment; patients were matched $1: 1$ by age, sex, thyroid nodule volume, nodule structure (solid), and thyroid function. A cumulative incidence function was used to describe regrowth trends and the Gray test to compare the cumulative incidence of regrowth between the RFA and LA groups. The Fine and Gray competing risk-regression model was used to identify predictors of regrowth over time. A univariate logistic=regression analysis was conducted to identify potential risk factors for technique inefficacy, regrowth, and retreatment. Receiver operating characteristic (ROC) studies were used to calculate the accuracy of volume, 1-year volume reduction, and energy as predictors of technique efficacy, regrowth, and retreatment.

\section{Results}

The study cohort was composed of 406 patients (median age, 57 years; range, 17-87; 75\% female). RFA was the treatment option in 216 patients (53\%), and 190 (47\%) were treated by LA. Patients with solid thyroid nodules represented $75 \%$ of the series, while $19 \%$ had a predominantly solid nodule, $5 \%$ had a predominantly cystic nodule, and $1 \%$ had a cyst. Only $9 \%$ of the patients had functional thyroid nodules. The median baseline nodule volume in the RFA group was $17.2 \mathrm{ml}$ (range, 0.4-179.0) and 12.2 $\mathrm{ml}$ (range, 1.7-86.0) in the LA group.

The median thyroid nodule volume-reduction rate (VRR) in the RFA group at 1, 2, 3, 4, and 5 years after the ablation was $72 \%, 75 \%, 76 \%, 76 \%$, and $77 \%$, respectively, and for the LA group, 55\%, 58\%, 59\%, $57 \%$, and $57 \%$. A total of $28 \%$ of patients ( 115 of 406 ) had thyroid nodule regrowth. A total of 79 patients (69\%) lost technique efficacy (the regrowth diminished the VRR to less than $50 \%$ ), $26 \%$ of patients (30 of 115) had relapse of symptoms, and $28 \%$ of patients (32 of 115) required retreatment. Regrowth was observed in $20 \%$ of patients treated with RFA (43 of 216 ) and in $38 \%$ of patients treated with LA (72 of 190); in the RFA group, $12 \%$ of patients (26 of 216) were retreated, while in the LA group, $24 \%$ of patients (46 of 190) were retreated. The only parameter that was independently associated with the risk of regrowth was the amount of energy delivered. Analyses of the cumulative incidence of nodule regrowth (and retreatment as the competing event) for a type of treatment showed that RFA was associated with a significantly lower risk of regrowth than LA.

A total of 46 of 406 patients (11\%) underwent thyroid surgery during the follow-up period, from which benign nodules were confirmed in 27 of 46 (59\%) and nonbenign pathology in 16 of 46 (35\%), while the final pathology results were missing in 3 (6\%). Patients in whom the thyroid nodule volume had decreased $<20 \%$ at 1 year after thermal ablative therapy were more likely to have nonbenign pathology according to the ROC analyses.

\section{Conclusions}

RFA and LA are increasingly popular thermal ablative therapies for the treatment of benign thyroid nodules, with both leading to a vital volume 

Nodules-The Role of Radio-Frequency Ablation and Laser Therapy

reduction that persists years after thermal ablation. However, in this multicenter cohort study, the risk of thyroid nodule regrowth was lower for the RFA technique than for LA, which may be attributable to the amount of energy delivered. It is important to note that regrowth occurred in less than one third of the patients overall, and most cases did not require further treatment. For patients whose nodule volume decreased $<20 \%$ at 1 year after thermal therapy, a repeat cytologic study or surgery should be considered owing to the increased risk of malignancy in such scenarios.

\section{COMMENTARY}

This study (4) demonstrates the vast experience of the Italian Minimally Invasive Treatments of the Thyroid Group in the thermal ablation therapies to treat benign thyroid nodules. There are some crucial concepts regarding the understanding of thermal ablation therapies. The first is to consider the volume of the thyroid nodule and not only its size in the greatest diameter. The second concept is that when we perform these treatments, we treat the patient's symptoms and not the nodule itself. This is important because, as demonstrated in this study, even though there may be a significant decrease of the volume of the thyroid nodule, in the vast majority of patients a relatively small nodule persists. Although some thyroid nodules showed partial regrowth, only a small proportion of these patients needed another treatment.

The main conclusion of the study is that RFA has superior results when compared to LA for the treatment of cytologically benign thyroid nodules. This conclusion is supported by other studies in the literature, as reported by Cho et al. (2) in a recent systematic review and meta-analysis. Another critical point to be commented on (that, unfortu- nately, this article does not address) and that has been very well detailed by Sim and Baek (3), would be the reasons for the regrowth of the nodule after therapy. Such reasons include the lack of treatment of the nodule's margins, the feeding artery, and the draining vein, the latter of which is usually located at the margin of the nodule.

Perhaps one of the reasons that could explain the superiority of RFA over LA seen in this study is the different procedures used to perform these two treatments. When performing RFA at my institution, as I have been doing for the last two years, we use a moving-shot technique with a trans-isthmic approach and hydrodissection $(1,5)$ that allows for better delivery of energy (4). However, when using LA, is applied the pullback technique with one to four fibers (5), which is less dynamic. Aside from the differences between these two techniques, my observation is that both are efficient, and the choice of the best method should be made according to local access to each technology, personal experience, and conditions of applicability. 
THYROID NODULES Thermal Treatment Options for Benign Thyroid Nodules-The Role of Radio-Frequency Ablation and Laser Therapy

Erivelto Volpi

\section{References}

1. Baek JH, Lee JH, Valcavi R, Pacella CM, Rhim H, Na DG 2011 Thermal ablation for benign thyroid nodules: Radiofrequency and laser. Korean J Radiol 12:525-540.

2. Cho SJ, Baek JH, Chung SR, Choi YJ, Lee JH 2020 Long-term results of thermal ablation of benign thyroid nodules: A systematic review and metaanalysis. Endocrinol Metab (Seoul) 35:339-350.

3. Sim JS, Baek JH 2019 Long-term outcomes following thermal ablation of benign thyroid nodules as an alternative to surgery: The importance of controlling regrowth. Endocrinol Metab (Seoul) 34:117-123.
4. Bernardi S, Giudici F, Cesareo R, Antonelli G, Cavallaro M, Deandrea M, Giusti M, Mormile A, Negro R, Palermo A, et al. 2020 Five-year results of radiofrequency and laser ablation of benign thyroid nodules: A multicenter study from the Italian Minimally Invasive Treatments of the Thyroid Group. Thyroid 30:1759-1770.

5. Cheng Z, Liang P 2020 Advances in ultrasoundguided thermal ablation for symptomatic benign thyroid nodules. Adv Clin Exp Med 29:1123-1129.

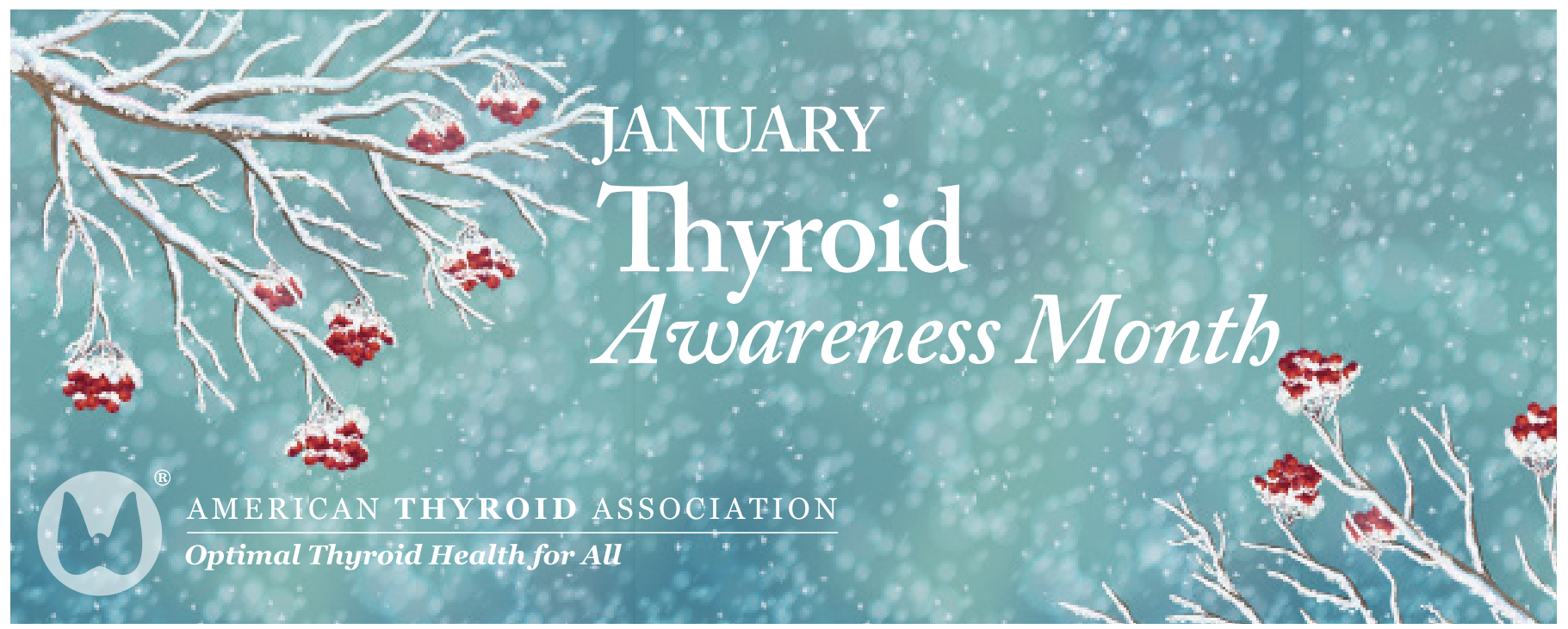

